Research Article

\title{
Case report: pigmented villinodular synovitis of both knee joints
}

\begin{abstract}
Pigmented villonodular synovitis (PVNS): Is a rare benign proliferative condition affecting synovial membranes of joints, bursae or tendons that are what we have seen in 47 years old women presented with longstanding painful swelling of both knees. Unknown etiology of the disease increases the suspicion of sharing other risk factors like trauma, Neutritional and psychological stresses. As It's useful to remind and list PVNS in a presumptive diagnosis of hemorrhagic, erosive and hemosiderine deposited arthritis we should consider it when we found the homogeneous hypo echogenic multi-nodular thickening of the synovium by sonography before trying the expensive and invasive diagnostic parameters.
\end{abstract}

Volume 10 Issue I - 2018

\author{
Uqba N Yousif \\ Department of medicine, Division of Rheumatology, Tikrit \\ University, Iraq
}
Correspondence: Uqba N Yousif, Department of medicine, Division of Rheumatology, Tikrit University, Iraq, Emailokynafy@yahoo.com

Received: December 16, 2017 | Published: January II, 2018

\section{Chief complaint}

47year old housewife female presented with long history of painful swelling of both knees.

\section{History of present illness}

The condition started before two years as simple intermittent swelling, pain and discomfort of the left knee joint responded to rest and simple analgesics. In the last 2 months the pain increased and both knees undergo sudden fulminant swelling. Left one was more than right. The condition aggravated with loss of function and inability to walk with intermittent locking. The provisional diagnosis by primary medical unit was Osteoarthritis treated by NSAID, exercises and physiotherapy but there was no response and the condition worsens and continued in deterioration without precise diagnosis till she visits our hospital.

\section{Systemic review}

No other musculoskeletal manifestation. No systemic Cardiopulmonary, Gastrointestinal, Central nervous system and skin findings.

\section{Past medical history}

Controlled hypertension, the patient had suffered from chronic knee joints pain and had no history of remarkable trauma or falls but she was walked a very long distances

Past surgical history: One cesarean section.

Family history: Negative.

Social history: She is married, 3 normal vaginal deliveries and one cesarean section, not alcoholic, not smoker. The patient had bad and difficult neutritional, psychological and physical situation.

Drug history: NSAID, Valsartan. No history of drug hyper sensitivity.

\section{Clinical examination}

A. Look; Left Knee swelling more than Right without any deformities.

B. Feel; Tenderness, Hotness. Thickened soft tissue of supra-patellar compartment of Left knee joint.

C. Movement; Mild Tenderness with limited range of movement ROM specially the Left one.
D. Special test; Patellar tap test positive in both knees but marked in Left one.

Investigations were done:

i. ESR 21.

ii. Rheumatoid factor was negative.

iii. ACCP Antibody negative.

iv. Normal CBC part of all other investigations.

v. Plain X ray of both knees was showing soft tissue swelling more in the left knee with normal bone contour \& mild joint space narrowing or grade I OA.

vi. Ultrasound report of knee joint: Complete \& real time exploration of Right and Left Knee.

Routine static and selective dynamic images were obtained in orthogonal plane. Normal gray scale sonographic findings of articular components.

vii. Left long knee supra patellar compartment finding:

i. Well marketed homogeneous echogenic irregular thickened glandular appearance of synovial tissue.

ii. Anechoic fluid appearance collection

No power Doppler signals intensity was shown. The Right Knee sonography reveals minimum amount of fluid with homogeneous hypo echoic thickened synovium.

viii. MRI: Left Knee typically shows mass like synovial proliferation with lobulated margins with low

signal intensity due to haemosiderin deposition. No bone involvement. Right Knee mild form of synovial proliferation without signals intensity.

ix. Arthrocentesis of the left knee: Aspiration about 25cc of bloody synovial fluid.

x. Synovial biopsy of the left knee report reveals abundant numbers of (pigmented) hemosiderin-laden macrophages with mononuclear histiocytes predominate mixed in with variable numbers of multinucleated giant cells (Figure $1 \& 2$ ). 


\section{Diagnosis}

Was made depending on all previous supportive diagnostic evidences as: Pigmented Villonodular Synovitis PVNS of both knee joints.

\section{Treatment}

After a failure of traditional therapeutic lines. Excisional subtotal synovectomy of the Left Knee joint was done then the patient feels better and following up the Right one.

\section{Discussion}

Pigmented villonodular synovitis (PVNS): Is a rare benign proliferative condition affecting synovial membranes of joints, bursae or tendons resulting from possibly neoplastic synovial proliferation with villous and nodular projections and haemosiderin deposition.

PVNS occurs predominantly in early to middle age $\left(2^{\text {nd }}\right.$ to $5^{\text {th }}$ decades). ${ }^{1,2}$ The exact cause of PVNS is unknown . There is no gender predilection, whereas extra-articular disease has a slight female predominance.

Unexplained joint swelling is disproportionate to the amount of pain that patient feels at first. Decreased motion and increased pain occur as the disorder progresses as well as locking of the joint. The patient often feels warm to the touch; actually that's what we have seen in our patient presenting symptoms. ${ }^{3}$

Pigmented villonodular synovitis, described distinctly in 1941 by Charles J Sutro, L Lichtenstein \& HL Jafe ${ }^{4}$ comes in two forms: localized and diffuse. Diffuse PVNS affects the entire synovium and sometimes develops in synovial lining of joints, tendon sheaths and bursae, almost always monoarticular and typically occurs in large joints such as the knee or hip and less commonly the ankles and shoulders. Also in little extents the spine might be involved. . $^{3,5,6}$ Although rare localized, or nodular, PVNS is less common than the diffuse form and typically occurs in smaller joints such as the hands and feet. Occasionally invades underlying bone \& may cause bone cyst formation, loss of bone and cartilage. ${ }^{6}$

We have found a hemorrhagic synovial fluid with features of chronic inflammatory change which is part of the disease behavior as mentioned by many authors. Some doctors believe it is caused by abnormal metabolism of fat. Others think it may be caused by repetitive inflammation. ${ }^{7,8}$

That's compatible with our patient who suffer from bad social situation with nutritionally, psychologically and physically exhausted along 2 years of walking a very long distanceson a mountainous and rugged surfaces bearing a different stresses on her knees was the reasonable predisposing factor resulted in repetitive inflammation.

Very little researches have been carried out. However, a common theme in patients is a trauma experienced to the joint prior to the onset of symptoms. ${ }^{9}$ It is locally aggressive and can spread to surrounding tissues, causing bone erosion and tissue damage. If not treated early, it can spread to areas outside the joint, and potentially cause permanent loss of range of movement as well as intense pain. ${ }^{10}$

We have used all clinical \& laboratory parameters of exclusion to rule out the diffuse PVNS symptoms which are often confused with those of Rheumatoid arthritis. ${ }^{11}$ PVNS is difficult to identify and is often not diagnosed for many years or more after presentation due to nonspecific symptoms or a general paucity of symptoms.
No definitive diagnostic test or universal diagnostic clinical criteria exists for PVNS a high index of suspicion is needed for diagnosis. A thorough medical history and physical examination are essential in addition to other non invasive affordable and feasible parameters like ultrasound assessment which shows a preliminary efficacy in diagnosis of this case.

The sonographic homogeneous echogenic hyper atrophic nodullar appearance of the synovium was one of the important attracting attention followed by joint aspiration of haemorragic fluid of patient denies any trauma that enhanced the thinking of diagnosis of PVNS.

Basically the treatment begins once PVNS is confirmed by biopsy of the synovium of an affected joint, a subtotal synovectomy of the affected area is the most common treatment and in this case the patient shows dramatic improvement regarding pain and joint function. Other studies mentioned that medical therapy is also being investigated in refractory cases including $\alpha$-TNF (tumour necrosis factor) blockade and infliximab. imatinib mesylate for the treatment of locally advanced form. ${ }^{12}$

Recurrence rates after subtotal synovectomy are reported to be $\sim 15 \%$ (range $7-20 \%$ ) (2)So radiation therapy may be considered as a treatment option. Sometimes Bone lesions might be caused by the disorder should be remove and bone grafting is performed as needed. In some cases, a total joint replacement is needed to relieve symptoms when PVNS causes significant joint destruction. ${ }^{13}$

\section{Conclusion \& Recommendations}

A. Since this disorder (PVNS) is rare \& difficult to identify and it is often not diagnosed for four years or more after presentation due to nonspecific symptoms or a general paucity of symptoms we need always not to forget and put it in our list of differential diagnosis of all huge synovial tissue thickening diseases, list of all hemorrhagic arthritis, list of haemosiderin deposition disease and list of all erosive arthropathy.

B. As a Fundamental rule PVNS is radiologically diagnosed by magnetic resonance imaging (MRI) and excisional biopsy. ${ }^{6}$ But in this case we have found that there was a guidance clue that increase the suspension of diagnosis of PVNS by using Musculoskeletal Ultrasound by the same hands of clinical examiner is rather helpful.

C. Trivial continues traumatic shocks on the joint due to certain work or walk or other different stresses such as Neutritional and psychological had a potential risk factor of PVNS and should not be forgotten in case history.

D. Further future studies should be dedicated on this subject thoroughly.

Play Video

Video 1: Left Transverse view of suprapatellar compartment

Play Video

Video 2: Left Longitudinal suprapatellar compartment

\section{Acknowledgements}

None.

\section{Conflicts of interest}

None. 


\section{References}

1. Kaplan P. Musculoskeletal MRI. W B Saunders Co, USA. 2001.।

2. Murphey MD, Rhee JH, Lewis RB, et al. Pigmented synovitis: radiologicpathologic correlation. Radiographics. 2008;28(5):1493-518.

3. John R McGrath, Felix S Chew, Johnny U V Monu. Pigmented Villonodular Synovitis Imaging. eMedicine. 2017.

4. Family Doctor.org: PVNS.

5. Parmar HA, Sitoh YY, Tan KK, et al. MR imaging features of pigmented villonodular synovitis of the cervical spine. AJNR Am J Neuroradiol. 2004;25(1):146-149.

6. Shankar V. Pigmented villonodullar synovitis (PVNS). 2017.

7. Mayclinic. Mayo Clinic.
8. Clinical Study. The Stone Clinic.

9. Jabalameli M, Jamshidi K, Radi M, et al. Surgical outcomes of 26 patients with pigmented villonodular synovitis (PVNS) of the knee at a mean follow-up of 4 years: Introducing a novel technique. Medical Journal of the Islamic Republic of Iran. 2014;28:123.

10. A Patient's Guide to Pigmented Villonodular Synovitis (PVNS) of the Knee.

11. Mayo Clinic.

12. Cassier PA, Gelderblom H, Stacchiotti S, et al. Efficacy of imatinib mesylate for the treatment of locally advanced and/or metastatic tenosynovial giant cell tumor/pigmented villonodular synovitis. Cancer. 2012;118(6):1649-1655.

13. https://www.medstarhealth.org/doctor/dr-frank-j-frassica-md/\#q $=\{\}$ 